\title{
RESECTION OF SINGLE BRAIN METASTASIS IN NON-SMALL-CELL LUNG CANCER: PROGNOSTIC FACTORS
}

\author{
Alfredo Mussi, $\mathrm{MD}^{\mathrm{a}}$ \\ Massimo Pistolesi, $\mathrm{MD}^{\mathrm{b}}$ \\ Marco Lucchi, MD $^{\mathrm{a}}$ \\ Alberto Janni, $\mathrm{MD}^{\mathrm{a}}$ \\ Antonio Chella, MD ${ }^{\mathrm{a}}$ \\ Giovanni Parenti, $\mathrm{MD}^{\mathrm{c}}$ \\ Giuseppe Rossi, $\mathrm{PhD}^{\mathrm{d}}$ \\ Carlo Alberto Angeletti, MD
}

\begin{abstract}
Combined resection of primary non-small-cell lung cancer and single brain metastasis is reportedly superior to other treatments in prolonging survival and disease-free interval. To identify prognostic factors that influenced survival we reviewed clinical records and follow-up data of 52 consecutive patients with non-small-cell lung cancer and single brain metastasis who had been evaluated for combined lung and brain operation: 19 had synchronous and 33 metachronous non-small-cell lung cancer and single brain metastasis. Seven patients were excluded from combined operation because of either early brain relapse after craniotomy or single brain metastasis localization in deep brain structures. Forty-one of the 45 patients who underwent combined operation had complete remission of neurologic symptoms. Actuarial 5-year survival from the second surgical intervention was $16 \%$ (median 19 months, range 1 to 104 months). N0 status and lobectomy were the only variables associated with longer survival. Actuarial 5-year survivals in patients with synchronous and metachronous presentation were $6.6 \%$ and $19 \%$, respectively. In patients with metachronous presentation the length of survival was significantly associated with N0 status, lobectomy, and interval between lung and brain operation equal to or longer than 14.5 months. The subset of patients with N0 status and interval between operations longer than 14.5 months had a 61\% 5-year survival. None of the patients with N1-2 disease and shorter interval between operations was alive at 20 months. These data indicate that prognostic factors may help to identify subsets of patients with markedly different outcomes after combined lung and brain operation. (J Thorac Cardiovasc Surg 1996;112:146-53)
\end{abstract}

$\mathrm{T}$ he brain is a common site of non-small-cell lung cancer (NSCLC) relapse. ${ }^{1}$ It has been estimated that in the United States about 40 thousand patients each year will have brain metastasis from NSCLC. ${ }^{2}$ Pathologic studies have shown that single brain

From the Servizio di Chirurgia Toracica, ${ }^{a}$ Dipartimento di Chirurgia, Universitá di Pisa; the Istituto di Clinica Medica II, ${ }^{b}$ Universitá di Pisa e Istituto di Fisiologia Clinica, CNR; the Istituto di Neurochirurgia, ${ }^{c}$ Universitá di Pisa; and the Reparto di Biostatistica ed Epidemiologia, ${ }^{\text {d }}$ Istituto di Fisiologia Clinica, CNR, Pisa, Italy.

Supported in part by the Ministry of University and Scientific and Technologic Research of Italy.

Received for publication June 26, 1995; accepted for publication Sept. 8, 1995.

Address for reprints: A. Mussi, MD, Servizio di Chirurgia Toracica, Dipartimento di Chirurgia, Via Roma 67, 56100 Pisa, Italy.

Copyright (C) 1996 by Mosby-Year Book, Inc.

0022-5223/96 $\$ 5.00+0 \quad \mathbf{1 2 / 1 / 6 9 2 5 1}$ metastasization occurs in one third of the whole population of patients with brain metastasis from NSCLC.$^{3-7}$ It is evident, therefore, that a considerable number of patients with NSCLC undergo evaluation for removal of single brain metastasis (SBM). It has been repeatedly demonstrated that combined operation of primary NSCLC and SBM is superior to other treatments in prolonging survival and disease-free interval. ${ }^{2,8-29}$ The aim of this paper was to ascertain whether survival could be affected by prognostic variables of both NSCLC and SBM and the time interval between the two surgical procedures.

\section{Patients and methods}

We reviewed the clinical records and follow-up data of 52 patients with NSCLC and SBM who, between January 1975 and June 1992, were evaluated for radical combined lung and brain operation.

In 19 patients the pulmonary and brain lesions were synchronous with neurologic symptoms as first clinical 
Table I. Clinical characteristics of 15 patients with synchronous presentation of lung cancer and SBM treated by combined operation

\begin{tabular}{lc}
\hline & $n$ \\
\hline Male/female & $15 / 0$ \\
Median age (yr) & 56.3 \\
Range (yr) & $41-68$ \\
& \\
Lung cancer & \\
Lobectomy/pneumonectomy & $13 / 2$ \\
Right/left site & $7 / 8$ \\
Upper/lower location & $12 / 3$ \\
Median size (cm) & 4 \\
Range (cm) & $2-9$ \\
Histologic type & \\
Squamous & 6 \\
Adenocarcinoma & 7 \\
Undifferentiated & 2 \\
T1-2/T3 status & $13 / 2$ \\
N0/N1-2 status & $8 / 7$ \\
Brain metastasis & \\
Right/left site & $10 / 5$ \\
Supratentorial/infratentorial location & $12 / 3$
\end{tabular}

$S B M$, Single brain metastasis.

presentation. Fifteen of these patients underwent combined brain and lung resections. Brain operation preceded lung resection in all cases (median interval 1 month) to control neurologic symptoms and to avoid central nervous system complications after pulmonary resection. The clinical characteristics of these patients are reported in Table I. Lung resection was not done in four patients because of early brain relapse after craniotomy.

In 33 patients SBM was diagnosed in a time interval equal to or longer than 2 months after NSCLC resection (metachronous presentation). Thirty of these patients underwent combined lung and brain resection with a median interval of 14.5 months (range 2 to 45 months). The clinical characteristics of these patients are reported in Table II. Brain resection was not done in three patients because of localization of the metastases in deep brain structures (medulla oblongata or basal ganglia).

The lung and brain resection was defined as complete according to standard rules. Margins of brain and lung resections were negative for neoplastic infiltration at microscopic examination. All the resected lung specimens were examined pathologically according to the TNM system. ${ }^{30}$ Differences between lung and brain histologic types were excluded by careful pathologic review of lung and brain neoplastic tissue of each patient.

Six of seven patients with synchronous presentation and N1-2 status and 5 of 11 patients with metachronous presentation and N1-2 status received various regimens of adjuvant chemotherapy after lung operation. Eight patients, two with synchronous and six with metachronous presentation, underwent whole brain irradiation with 30 Gy in 15 fractions over 4 weeks. Three patients with T3 tumors and metachronous presentation of SBM underwent chest wall radiotherapy after en bloc resection.
Table II. Clinical characteristics of 30 patients with metachronous presentation of lung cancer and SBM treated by lung and subsequent brain operation

\begin{tabular}{lc}
\hline & $n$ \\
\hline Male/female & $26 / 4$ \\
Median age (yr) & 57.5 \\
Range (yr) & $33-72$ \\
& \\
Lung cancer & \\
Lobectomy/pneumonectomy & $22 / 8$ \\
Right/left site & $12 / 18$ \\
Upper/lower location & $26 / 4$ \\
Median size (cm) & 4 \\
Range (cm) & $2-10$ \\
Histologic type & \\
Squamous & 9 \\
Adenocarcinoma & 12 \\
Undifferentiated & 9 \\
T1-2/T3 status & $22 / 8$ \\
N0/N1-2 status & $19 / 11$ \\
Brain metastasis & \\
Median interval (mo) & 14.5 \\
Range (mo) & $2-45$ \\
Right/left site & $16 / 14$ \\
Supratentorial/infratentorial location & $24 / 6$ \\
\hline
\end{tabular}

$S B M$, Single brain metastasis.

Survival time was measured from the date of the second surgical procedure (lung for patients with synchronous presentation and brain for patients with metachronous presentation) until death or the most-recent date of follow-up (December 1993) for those surviving. Survival was estimated by the Kaplan-Meier method ${ }^{31}$ and comparisons of survival for univariate analysis were estimated by Mantel-Cox and Breslow tests. ${ }^{32,33}$ Frequency data analysis was estimated by Fisher's exact test. The results were considered significant at the 0.05 level $(p<0.05)$.

\section{Results}

There were no operative deaths. Complete remission of the neurologic symptoms was obtained in 41 of the 45 patients who underwent combined operation. The actuarial overall 5-year survival was $16 \%$ with a median survival of 19 months (range 1 to 104 months) (Fig. 1). Thirty-six patients had relapse and died of the tumor. Table III reports sites of relapse and disease-free interval from the second surgical intervention. A 77-year-old patient with a 47-month disease-free interval from time of brain operation died of cerebral ictus. By the univariate model (Table IV), the following did not affect survival: sex; age; site, size, location, histologic type, and $\mathrm{T}$ status of lung cancer; site and location of SBM; synchronous or metachronous presentation; and adjuvant therapy. By contrast, survival was significantly af- 


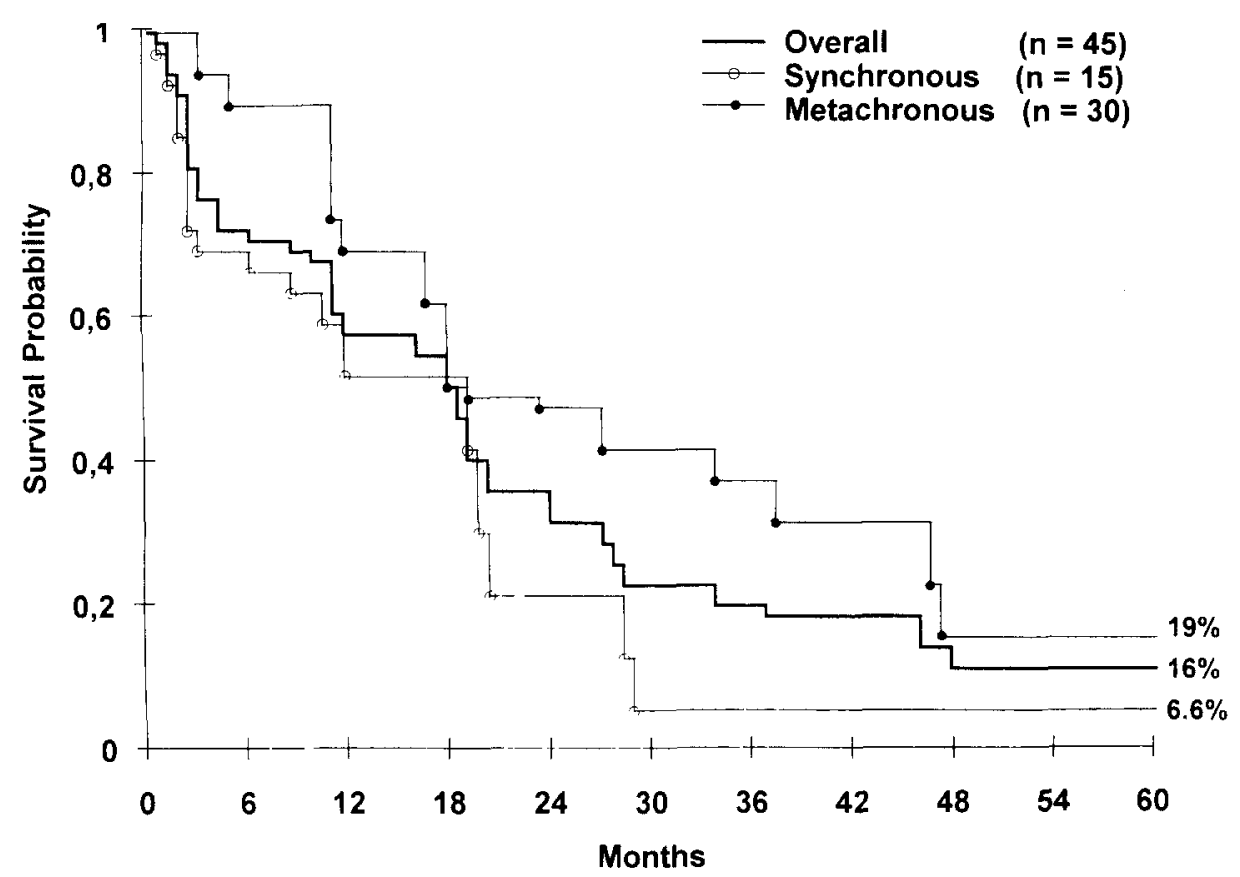

Fig. 1. Actuarial 5-year survival of all patients treated by combined lung and brain operation. Median survival was 19 months for all patients. Patients with synchronous and metachronous presentations had median survivals of 18 and 19 months, respectively.

Table III. Site of relapse and disease-free interval of 36 patients treated by combined lung and brain operation who had relapse

\begin{tabular}{ccc}
\hline $\begin{array}{c}\text { Site of } \\
\text { relapse }\end{array}$ & $n(\%)$ & $\begin{array}{c}\text { Median } \\
\text { disease-free interval } \\
\text { in months (range) }\end{array}$ \\
\hline Local & & \\
Thorax & $14(39)$ & $13.5(2-95)$ \\
Brain & $8(22)$ & $14.5(2-23)$ \\
Systemic & $14(39)$ & $2(1-54)$ \\
\hline
\end{tabular}

fected by type of lung resection (lobectomy versus pneumonectomy) (Fig. 2) and N status (N0 versus N1-2) (Fig. 3).

The 5-year survival rate of the 15 patients with synchronous presentation was $6.6 \%$ with a median survival of 18 months (Fig. 1). Fourteen of the 15 patients died within 30 months. Only one patient survived more than 5 years (63 months). The only variable that was significantly associated with a longer survival was the presence of squamous lung cancer $(p=0.02)$.

The 5-year actuarial survival of the 30 patients with metachronous presentation was $19 \%$ with a median survival of 19 months (Fig. 1). The variables
Table IV. Univariate analysis of 45 patients with lung cancer and SBM (synchronous and metachronous) treated by combined operation

\begin{tabular}{lc}
\multicolumn{1}{c}{ Variables } & $p$ Value \\
\hline Male vs female & NS \\
Age $<57$ vs $\geq 57$ yr & NS \\
& \\
Lung cancer & \\
Lobectomy vs pneumonectomy & 0.040 \\
Right vs left site & NS \\
Size $<4$ vs $\geq 4$ cm & NS \\
Upper vs lower location & NS \\
Squamous vs nonsquamous histologic type & NS \\
T1-2 vs T3 status & NS \\
N0 vs N1-2 status & 0.009 \\
Brain metastatis & \\
Synchronous vs metachronous presentation & NS \\
Right vs left site & NS \\
Supratentorial vs infratentorial location & NS \\
Adjuvant therapy & NS \\
\hline
\end{tabular}

NS, Not significant; $S B M$, single brain metastasis.

associated with a longer survival (Table V) were the type of lung resection (median survival of patients who underwent lobectomy and pneumonectomy was 27 and 4 months, respectively), the $\mathrm{N}$ status (median survival of patients with N0 and N1-2 tumors was 37 


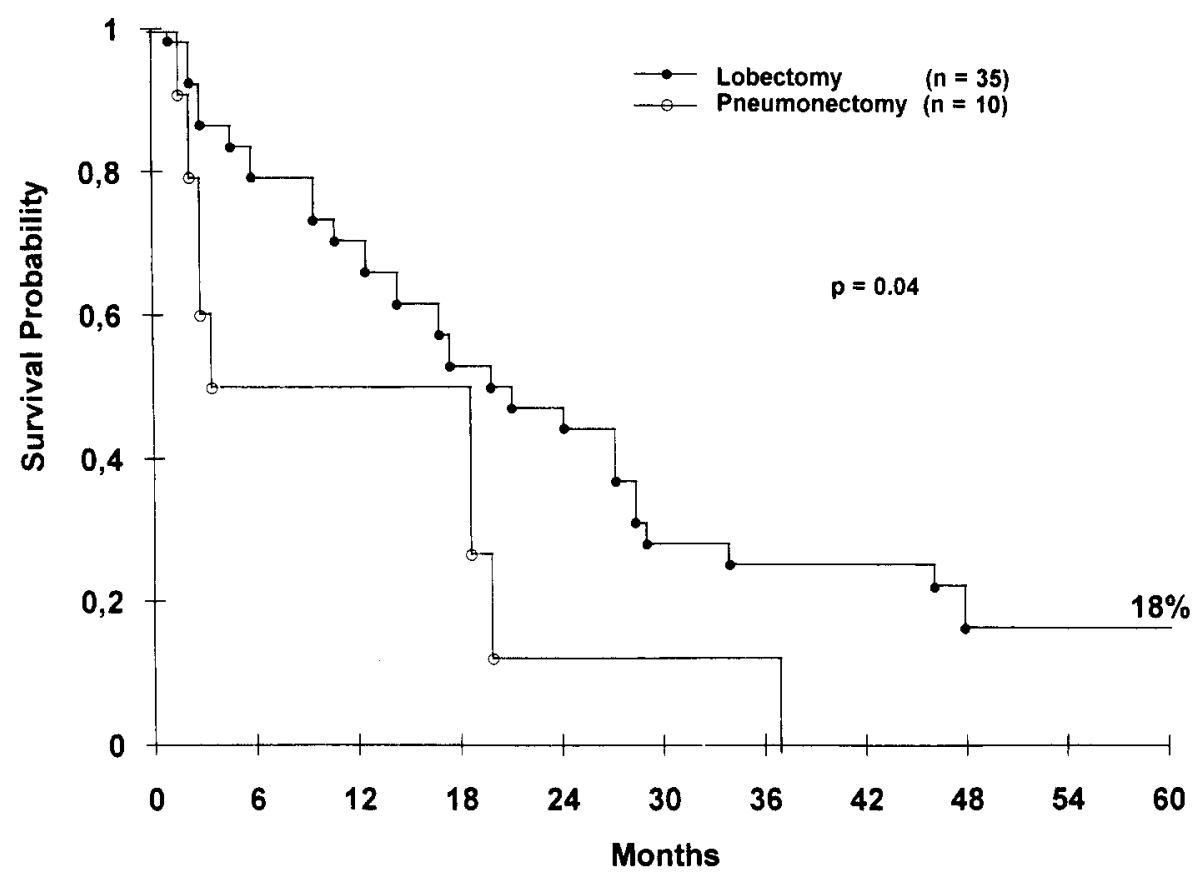

Fig. 2. Actuarial 5-year survival according to type of lung operation in 45 patients who underwent combined lung and brain operation. Median survival of patients who underwent lobectomy and pneumonectomy was 20 and 5 months, respectively.

and 4 months, respectively), and the median interval between lung and brain operation ( $\geq 14.5$ months versus $<14.5$ months) (Fig. 4 ).

With grouping of the 30 patients with metachronous presentation according to $\mathrm{N}$ status and interval between the two surgical procedures, the longest 5 -year survival $(61 \%)$ was obtained in patients with No status and interval between lung and brain operation equal to or longer than 14.5 months $(p=$ 0.004 ) (Fig. 5). All patients with N1-2 status and interval between surgical procedures shorter than 14.5 months died within 20 months. Combining $\mathrm{N}$ status with type of lung operation, there was $29 \%$ 5 -year survival in the 14 patients with N0 status and lobectomy. Only one of the other 16 patients was alive at 5 years. Although statistical significance in survival was not reached among the small subgroups of patients combined according to type of lung intervention and interval between surgical procedures, the 12 patients who underwent SBM removal after a period equal to or longer than 14.5 months after a lobectomy had an actuarial 5-year survival of $58 \%$. On the contrary, none of the remaining 18 patients was alive after 4 years.

\section{Discussion}

The results of this paper confirm previous data of the literature that show that combined lung and brain operation is an effective treatment to control symptoms and to prolong survival in patients with NSCLC and SBM. ${ }^{2,8-29}$ Indeed, in the present series of patients the median survival was 19 months, whereas the reported median survival in untreated patients or in those treated with other therapeutic modalities does not exceed 6 months. ${ }^{2}$

Furthermore, the present data indicate that survival is greatly affected by the locoregional extension of the lung tumor. In fact, considering the whole population of patients, type of lung resection and $\mathrm{N}$ status were associated with significantly different survival probabilities. Survival was higher in patients who underwent lobectomy and in those who had NO status with respect to survival in patients who underwent pneumonectomy and those with N1-2 status.

The other variables considered, including synchronous or metachronous presentation, did not attain significant differences as far as survival probability was concerned. However, the survival at 5 


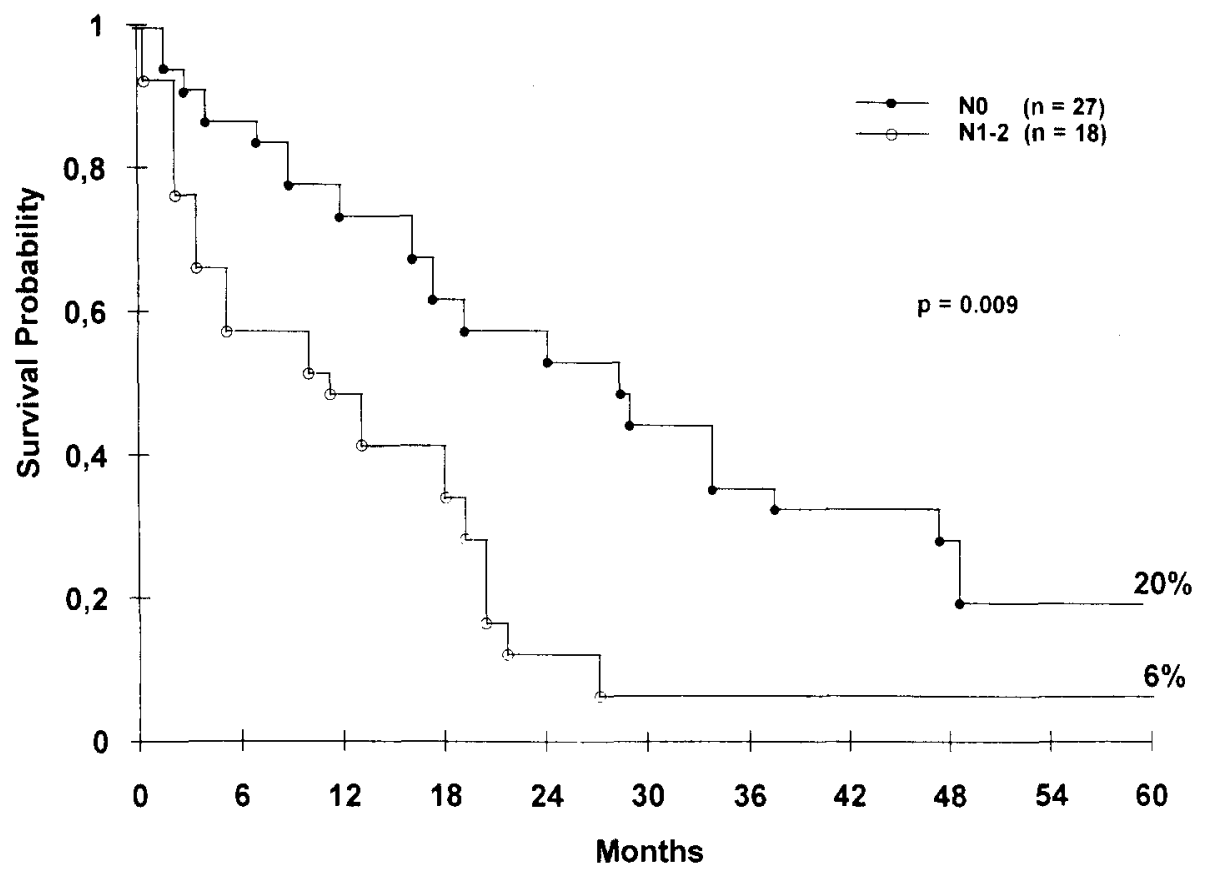

Fig. 3. Actuarial 5-year survival according to $\mathrm{N}$ stage considering 45 patients who underwent combined lung and brain operation. Median survival for N0 and N1-2 tumors was 28 and 12 months, respectively.

Table V. Univariate analysis of 30 patients with metachronous presentation of lung cancer and SBM treated by combined operation

\begin{tabular}{lc}
\multicolumn{1}{c}{ Variables } & $p$ Value \\
\hline Male vs female & NS \\
Age $<57$ vs $\geq 57 \mathrm{yr}$ & $\mathrm{NS}$ \\
& \\
Lung cancer & 0.024 \\
Lobectomy vs pneumonectomy & $\mathrm{NS}$ \\
Right vs left site & $\mathrm{NS}$ \\
Size $<4$ vs $\geq 4 \mathrm{~cm}$ & $\mathrm{NS}$ \\
Upper vs lower location & $\mathrm{NS}$ \\
Squamous vs nonsquamous histologic type & $\mathrm{NS}$ \\
T1-2 vs T3 status & 0.026 \\
N0 vs N1-2 status & \\
Brain metastatis & $\mathrm{NS}$ \\
Right vs left site & $\mathrm{NS}$ \\
Supratentorial vs infratentorial location & 0.045 \\
Interval between treatments less than median & \\
or greater than or equal to median & \\
$\quad$ (14.5 mo) &
\end{tabular}

NS, Not significant; $S B M$, single brain metastasis.

years was significantly higher $(p=0.007)$ in patients with metachronous presentation (31\%, median 35 months) with respect to survival in patients with synchronous presentation $(6.6 \%$, median 18 months) when the time lapse from diagnosis of
NSCLC to death or the date of the most-recent follow-up was considered for the computation, instead of the time from the second surgical intervention. The observation that a metachronous presentation could represent a favorable indication for prognosis was further suggested by the results obtained in the analysis of the prognostic factors in the 30 patients with metachronous presentation. Indeed, a significant increase in survival probability was associated not only with a limited extension of NSCLC (lobectomy versus pneumonectomy and N0 versus N1-2), but also with the length of the median interval between the two surgical procedures $(\geq 14.5$ months).

Furthermore, the presence of more than one favorable prognostic factor in patients with metachronous presentation was associated with an even longer survival. As an example, patients with N0 status and an interval between interventions equal to or longer than 14.5 months had a markedly different prognosis (median 34 months) with respect to the prognosis in patients with N1-2 status and a shorter interval (median 4 months). Although based on small subsets of patients, this observation points to the relevance of the previously mentioned prognostic indicators to the therapeutic decision. Indeed, patients with poor prognostic 


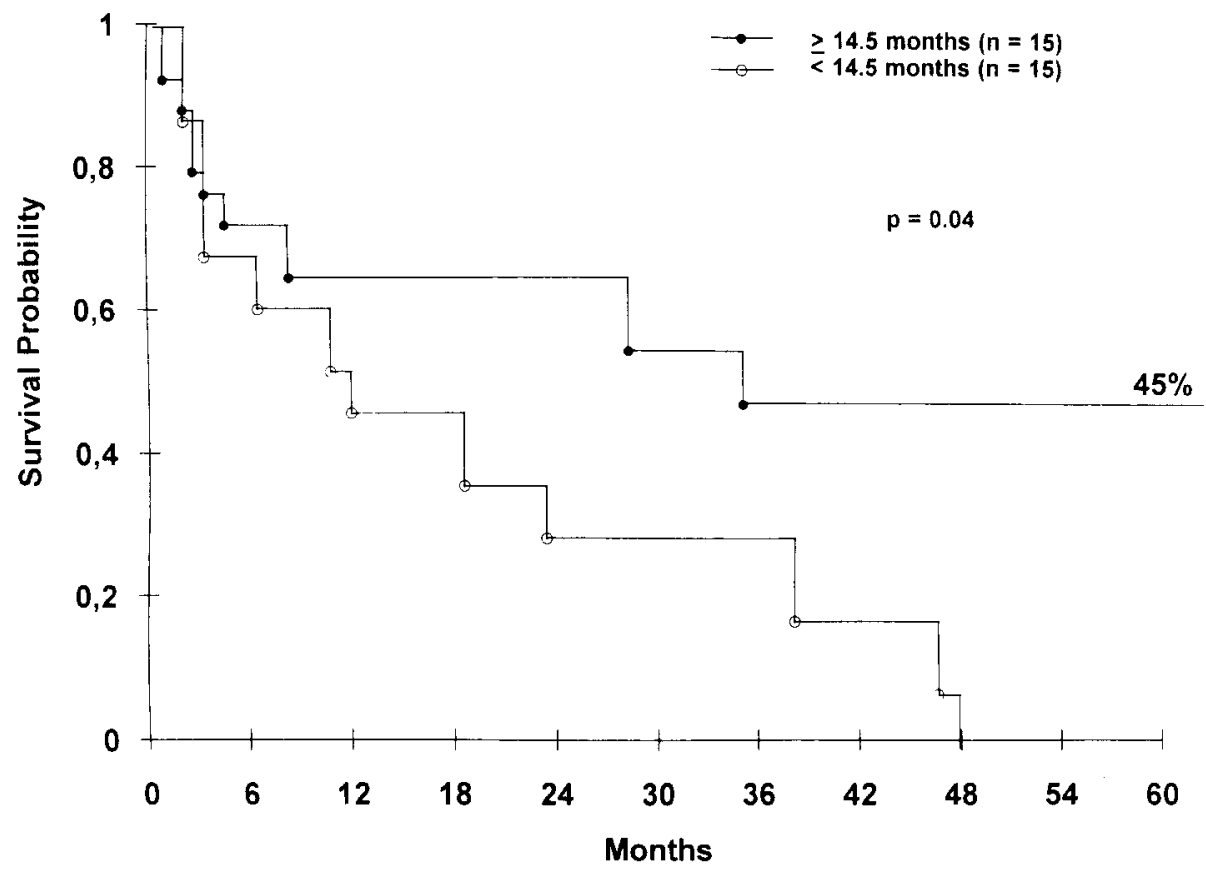

Fig. 4. Actuarial 5-year survival according to interval between lung and brain operation in 30 patients with metachronous presentation. Median survival of patients with interval between treatments 14.5 months or longer was 34 months compared with 12 months for patients with interval less than 14.5 months.

factors will probably not receive great benefit from the removal of SBM.

In the patients with synchronous presentation no definite prognostic factors were identified with the exception of a trend to a longer survival in patients with squamous cell carcinoma.

As concerns adjuvant whole brain irradiation, Magilligan, ${ }^{17}$ Catinella, ${ }^{26}$ and Hankins ${ }^{34}$ and their associates showed a beneficial effect, whereas Burt and associates ${ }^{2}$ did not find any significant increase in survival after postoperative brain irradiation. In a randomized study Patchell and colleagues ${ }^{35}$ demonstrated that patients who underwent operation plus whole brain irradiation had significantly longer survival in comparison with patients treated only with whole brain irradiation. Our results based on a small number of patients who received adjuvant whole brain irradiation do not permit us to derive firm conclusions about the usefulness of this therapeutic modality. However, in the present study, the great majority of patients died as a consequence of extracranial relapse of the tumor. This points to the superior relevance of the lung tumor local stage, as compared with that of the SBM, in determining the prognosis of these patients. This is further evidenced in patients with metachronous presentation who at the time of the most-recent follow-up showed only a $13 \%$ rate of brain relapse. Given the high morbidity and the undesirable effects connected with whole brain irradiation, it may be advisable to limit this treatment to selected cases.

The small percentage of cases in which the patient underwent adjuvant chemotherapy and the different therapeutic regimens adopted at different times during the course of the study do not permit us to derive general conclusions on this subject.

The observation of an association of the length of survival with the local stage of the lung tumor in the whole population of patients, as well as with the length of the interval from lung operation to brain relapse in patients with metachronous presentation, permits us to draw some considerations on the therapeutic approach to these patients with dismal prognoses. Although based on a small number of subjects, the results obtained in this study clearly indicate that the removal of SBM in patients with NSCLC has to be considered after accurate evaluation of the prognostic factors involved. With an accuracy reasonably acceptable from the clinical point of view, great differences in the outcome can be anticipated in each patient. In general, it can be said that there are patients in whom removal of 


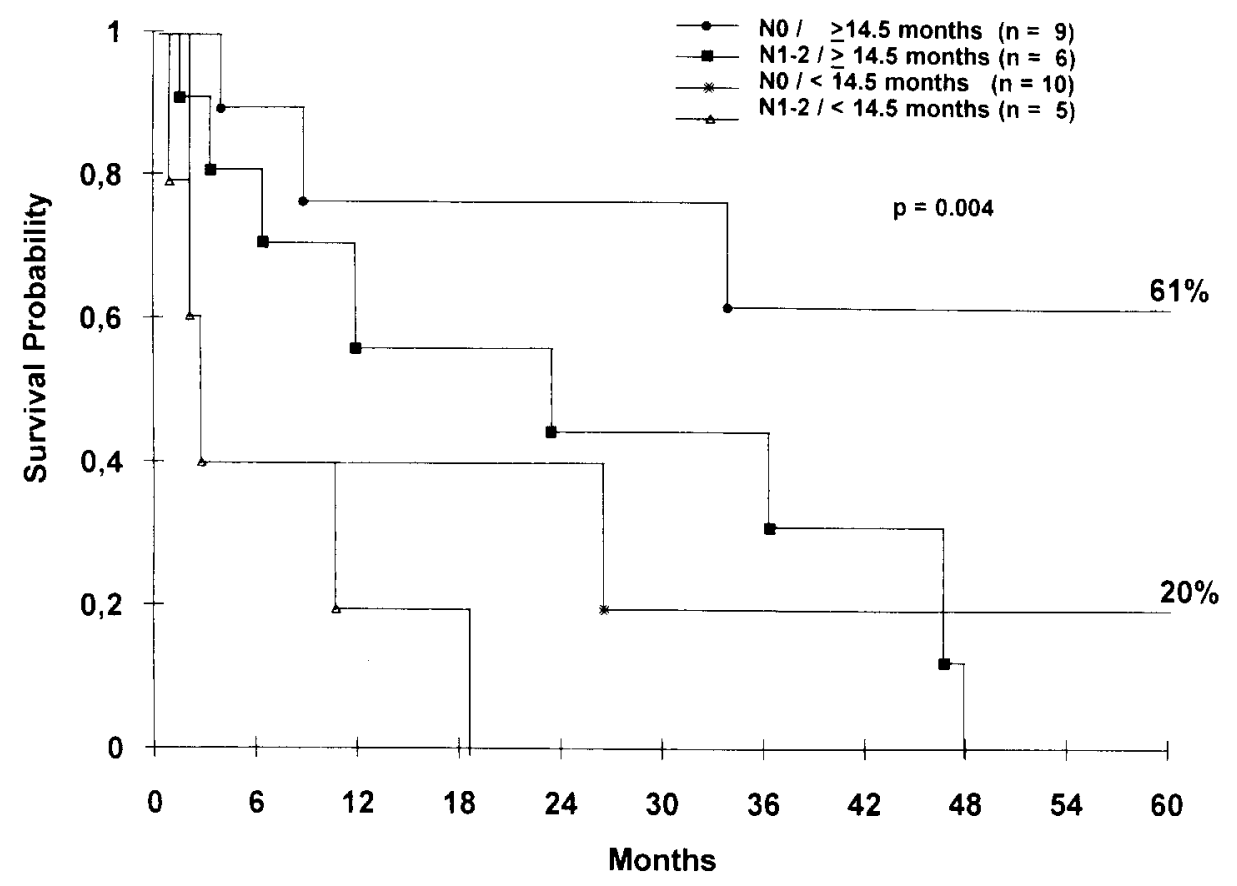

Fig. 5. Actuarial 5-year survival of patients with metachronous presentation of SBM stratified according to $\mathrm{N}$ stage and interval between lung and brain operation. Median survival was 34 months for N0, 14.5 months or longer; 4 months for N1-2, 14.5 months or longer; 24 months for N0, less than 14.5 months; and 4 months for N1-2, less than 14.5 months.

SBM could be viewed only as an effective means for symptomatic relief. This is particularly true for patients with locally advanced lung tumors, patients with synchronous presentation, and patients with a short interval between lung operation and brain relapse. In the latter group the observed short-term survival after brain operation is not significantly different from that obtainable with alternative therapies. For this reason, removal of SBM in this subgroup of patients is warranted only in accurately selected cases. By contrast, the excellent results obtained in patients with limited locoregional NSCLC involvement and a long interval between lung operation and brain relapse point to a potential curative effect of SBM removal. This subgroup of patients demonstrates long-term survival by brain operation, a result that cannot be achieved by other treatments.

That a limited locoregional stage of the lung tumor had a favorable bearing on the outcome has been already observed by others. ${ }^{17,21,24,25}$ In agreement with this, Burt and associates ${ }^{2}$ found that patients with complete resection of NSCLC survived significantly longer than those with residual locoregional disease. However, comparison of our data with those of Burt and associates ${ }^{2}$ is not possible because those authors did not report an analysis of the prognostic factors in the subgroup of patients who had completely resectable locoregional disease.

In conclusion, the data obtained provide further support to the therapeutic approach of combined brain and lung operation in patients with NSCLC and SBM and, furthermore, indicate that accurate disease staging and selection of patients may help identify subsets of patients who will obtain the greater benefit from this procedure in terms of length of survival. Confirmation of these results in a greater study population made of larger subsets of patients with different prognostic characteristics is warranted to better define advantages and limits of this therapeutic procedure.

\section{REFERENCES}

1. Newman SJ, Hansen HH. Frequency, diagnosis and treatment of brain metastasis in 247 consecutive patients with bronchogenic carcinoma. Cancer 1974;33:492-6.

2. Burt M, Wronski M, Arbit E, Galicich JH, and The Memorial Sloan-Kettering Cancer Treatment Thoracic Surgical Staff. Resection of brain metastases from non-small-cell carcinoma: results of therapy. J Thorac Cardiovase Surg 1992;103: 399.410 .

3. King AB, Ford FR. A clinical and anatomical study of 
neurological conditions resulting from metastases in the central nervous system due to carcinoma of the lung: review of 100 cases. Bull Johns Hopkins Hosp 1942;70:124-56.

4. Flavel G. Solitary cerebral metastases from bronchial carcinoma. BMJ 1949;2:736-7.

5. Knights EM Jr. Metastatic tumors of the brain and their relation to primary and secondary pulmonary cancer. Cancer 1954;7:259-65.

6. Galluzzi S, Payne PM. Brain metastases from primary bronchial carcinoma: a statistical study of 741 necropsies. Biol J Cancer 1956;10:408-24.

7. Richards $P$, McKissock W. Intracranial metastasis. BMJ 1963;1:15-8.

8. Oldberg E. Surgical considerations of carcinoma metastases to the brain. JAMA $1933 ; 101: 1458$.

9. Bakay L. Results of surgical treatment of intracranial metastasis from pulmonary cancer: report of a case with 5 years survival. J Neurosurg 1958;15:338-41.

10. Simionescu MD. Metastatic tumors of the brain: a follow-up study of 195 patients with neurosurgical considerations. J Neurosurg 1960;17:361-73.

11. Veight RG, Odom GL. Intracranial metastases and their surgical treatment. J Neurosurg 1965;23:375-83.

12. Stoier M. Metastatic tumors of the brain. Acta Neurol Scand 1965;41:262-78.

13. MacGee EF. Surgical treatment of cerebral metastases from lung cancer: the effect on quality and duration of survival. J Neurosurg 1971;35:416-20.

14. Hendricks GL Jr, Barnes WT, Hood HL. Seven year "cure" of lung cancer metastases to the brain. JAMA 1972;220:127.

15. Mosberg WH Jr. Twelve year "cure" of lung cancer metastases to brain. JAMA 1976;235:2745-6.

16. Tarmoff JE, Calinog TA, Byla JG. Prolonged survival following cerebral metastasis from pulmonary cancer. J Thorac Cardiovasc Surg 1976;72:933-7.

17. Magilligan DJ, Rogers JS, Knighton RS, Davila JC. Pulmonary neoplasm with solitary cerebral metastasis: results of combined excision. J Thorac Cardiovasc Surg 1976;72:690-8.

18. Salerno TA, Little JR, Munro DD. Bronchogenic carcinoma with a brain metastasis: a continuing challenge. Ann Thorac Surg 1979;27:235-7.

19. Zimm S, Wampler GL, Stablein D, Hazra T, Young HF. Intracerebral metastases in solid tumor patients: natural history and results of treatment. Cancer 1981;48:384-94.

20. Creig NH. Chemotherapy of brain metastasis: current status. Cancer Treat Rev 1984:11:157-86.
21. Sundaresin N, Galicich JH. Surgical treatment of single brain metastases from non small cell lung cancer. Cancer Invest 1985;3:107-13.

22. Mussi A, Janni A, Pistolesi M, Ravelli V, Buonaguidi R, Angeletti CA. Surgical treatment of primary lung cancer and solitary brain metastasis. Thorax 1985;40:191-3.

23. Mandel L, Hilaris B, Sullivan M, et al. The treatment of single brain metastasis from non oat cell lung carcinoma. Cancer 1986;58:641-9.

24. Magilligan DJ. Treatment of lung cancer metastatic to the brain. Surg Clin North Am 1987;5:1073-80.

25. Torre M, Barbieri B, Bera E, LoCicero S, Nerli FR, Belloni PA. Surgical therapy in lung cancer with single brain metastasis. Eur J Cardiothorac Surg 1988;2:336-9.

26. Catinella F, Kittle CF, Faber LP, Milloy FJ, Warren WH, Von Roenn KA. Surgical treatment of primary lung cancer and solitary intracranial metastasis. Chest 1989;95: 972-5.

27. Read RC, Boop WC, Yoder G, Schaefer R. Management of non-small-cell lung carcinoma with solitary brain metastasis. J Thorac Cardiovasc Surg 1989;72:690-8.

28. Macchiarini P, Buonaguidi R, Hardin M, Mussi A, Angeletti $\mathrm{CA}$. Results and prognostic factors of surgery in the management of non small cell lung cancer with solitary brain metastasis. Cancer 1991;68:300-4.

29. Chang DB, Yang PC, Luh KT, Kuo SH, Hong RL, Lee LN. Late survival of non small cell lung cancer patients with brain metastases: influence of treatment. Chest 1992;101: 1293-7.

30. Mountain C. A new international staging system for lung cancer. Chest 1986;89:225-33.

31. Kaplan EL, Meier P. Nonparametric estimation from incomplete observations. J Am Stat Assoc 1958;53:457-81.

32. Peto $R$, Pike MC, Armitage P, Breslow NE, Cox DR, Howard SY. Design and analysis of randomized clinical trials requiring prolonged observation of each patient: analysis and examples. Br J Cancer 1977;35:1-39.

33. Cox DR. Regression models in life tables. J R Stat Soc (B) 1972;34:187-220.

34. Hankins JR, Miller JE, Sallman M, et al. Surgical management of lung cancer with solitary cerebral metastasis. Ann Thorac Surg 1988;46:24-8.

35. Patchell RA, Tibbs PA, Walsh JW, et al. A randomized trial of surgery in the treatment of single metastases to the brain. N Engl J Med 1990;322:494-500. 